
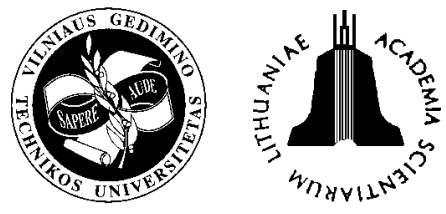

\title{
A MATHEMATICAL MODEL FOR EVALUATING ACCELERATED WEAR DUE TO INEFFICIENT MAINTENANCE
}

\author{
Sunday Ayoola Oke, Ayokunle Bamigbaiye, Oluwafemi Isaac Oyedokun \\ Department of Mechanical Engineering, University of Lagos, Akoka-Lagos, Nigeria, \\ E-mail:sa_oke@yahoo.com
}

Received 5 December 2005; accepted 28 March 2006

\begin{abstract}
This paper examines accelerated wear due to inefficient maintenance practice in the engineering organization, with a special focus on plant machinery. It considers some important parameters, mathematical relations and how to reduce the effect of poor maintenance practices on plant machinery. Mathematical tools of calculus, series, statistics, and variation were utilized in the development of the model. The result shows that poor maintenance practices increase accelerated wear in plant machinery while good maintenance is aided by planned maintenance, well-stocked inventory of spare parts, good production procedures, extended plant life, etc. This work is limited in scope since it considers a limited number of parameters as measures of wear. The paper is valuable to the maintenance manager and practitioners who intend to produce/manufacture and at the same time minimize the accelerated wear in production machinery by close monitoring, and using the model as a tool. The work is of immense benefits to managers in process industries, particularly in oil and gas exploration activities. The work is new, presenting a simple approach for easy adaptation in the industry. The approach utilized is not yet documented in the maintenance engineering literature. Thus, a new area of research is opened up for research explorations for the members of the maintenance engineering community.
\end{abstract}

Keywords: wear rate, maintenance engineering, inefficient maintenance, manufacturing system, accelerated wear.

\section{Introduction}

Manufacturing generally involves the combination of tools, equipment, and processes to convert raw materials into a finished product through the most efficient and cost-effective means [1,2]. Manufacturing, however, will either be very slow and inefficient or altogether impossible without the use of machines. These machines are usually mechanical devices with several mechanisms to create the effects of a motion or power transmission from one point to another. Other mechanisms include the conversion of one motion to another (e.g. rotational to reciprocating), increasing the speed of motion, changing the direction of rotation or even the plane of a motion.

Machines in operation generally involve mechanical actions of bodies (linkages) moving relative to one another. This causes heat to be generated, thus resulting in the removal of layers of machine parts in a relative motion. Although this seems natural in plant machinery maintenance, its effects could take alarming dimensions when the maintenance practice designed to minimize wear is poorly done; hence we have accelerated wear [3, 4]. Maintenance, which is a set of activities aimed at ensuring that plants operate optimally can be broadly classified into two: planned and unplanned maintenance. Accelerated wear is reduced by operating planned maintenance while unplanned maintenance is a corrective measure aimed at restoring broken down machines (plant parts) to normal operating capacity.

Accelerated wear among other effects, leads to the reduction of plant life, unexpected machine breakdown (shut downs), breaks in the production chain, failure to meet production targets, disappointment of company customers, loss of patronage through brand switching (change in loyalty of one's customer to other more reliable operators within the same business), decline in profit, and consequently, the growth of the business [5-7]. This model considers several measures of wear i.e. parameters used to evaluate the plant-accelerated wear). These include skill of the operators of the plant machinery (expertise), duration of plant operation, wear function of each machine, the leakage rate (that is, the rate of loss of liquid from the piping system due to leaks). Since the life of the plant 
depends on the wear rate, it is of the utmost importance to undertake this study on accelerated wear as a function of maintenance practice [6-8].

The paper is organized as follows: Section 2 discusses the methodology used while presenting the framework under a number of stated assumptions. In section 3 , a case study is provided in order to illustrate the practical relevance of the model proposed in section 2. Section 4 presents the analysis of results while in section 5 , the conclusions are presented with a brief discussion of the lessons learnt and future extension of this study.

\section{Methodology}

\subsection{The theoretical framework}

The mathematical theories applied in the model are calculus, statistics, arithmetic sequence and variation. Variation was used in the model design so that wear and other parameters such as a manpower factor, volume rate of fluid cost from joints, etc. can be related and expressed mathematically. The wear parameters are represented as continuous functions of time. The motivation for this is that time is an essential parameter on which other parameters depend. Making a function continuous makes it easier to work with as mathematical operations of differentiation and integration over duration will be carried out easily. Another important reason for utilizing calculus is that parametric variation (say with time) is made possible and useful as a measure of wear using calculus.

The mean of a particular variable is at times obtained so as to get a representative value for all the data at hand. This is a statistical tool. The machinery in a plant will wear at different rates due to different conditions and duration of use that they are exposed to. By taking the mean we would have more meaningful representation of the wear for the whole plant than using total wear. The wear in each machine is also not fixed; it can be expressed as a continuous function of time (this is called the machine wear function fit). It enables flexibility in the mathematical behaviour of each machine run over the same period of time. The mean of the wear obtained is then taken as a representative value for the plant wear.

The motivation for utilizing an arithmetic sequence as a tool for the model design is that for part A of the analysis, it is assumed that even when the plant is given planned maintenance, operated as specified by the manufacturer, loaded appropriately, it will still wear steadily. It wears by amount $(\Delta w)$ which value will later be assumed for every $t$ hour day of plant operation.

If for the first day of plant operation ( $t$ hours), the wear is $w_{0}$, then, for the next day it will be
$w_{0}+\Delta w$, the $3^{\text {rd }}$ day it will be $\left(w_{0}+\Delta w\right)+\Delta w=w_{0}+2 \Delta w$. For the $4^{\text {th }}$ day, the plant wear will be $w_{0}+3 \Delta w$.

Therefore, for $n$ days of plant operation, we will have $w_{0}+(n-1)(\Delta w)$ as the amount of wear. This is similar to the formula for the $n^{\text {th }}$ term of an arithmetic sequence which first term is $a$, having common difference $d$ and $a$ value:

$$
\tau_{n}=a+(n-1) d \text {. }
$$

$$
\begin{aligned}
& \text { Also, } \\
& w_{n}=w_{0}+(n-1)(\Delta w) .
\end{aligned}
$$

By comparing equations (1) and (2), we note that $w_{n}=\tau_{n}$; and $a=w_{0}$. Also, $(n-1)$ is the number of days of plant operation. We also note that $\Delta w=$ additional wear due to a day of plant operation, $w_{n}=$ wear in the machine after $n$ days of operation.

$w_{0}$ is made different from the incremental value of the wear $(\Delta w)$ so that the initial wear on the plant can, if desired, be made a value different from that of incremental wear $(\Delta w)$. The justification for this approach is that machinery always has a measure of friction no matter how well maintained. This has wear associated with it. Moreover, using an arithmetic sequence to represent it will give the slowest wear rate (which is desired as a mathematical representation/ model for good maintenance).

Using of geometric sequence, exponential function, or similar mathematical tools will give wear values that will build up rapidly. This will make it impossible to merge the concept (good maintenance) and the mathematical implication (slow wear rate) for planned maintenance.

\subsection{Assumptions}

It may be difficult to find a very effective way of measuring wear in a plant in view of the complexity involved. Many machines operating on different principles consequently will exhibit wear by different means. The effect of plant acquisition or downsizing on the wear measurement put in place, maintenance (planned and unplanned), shut downs, changes in prevailing technology of production, etc. makes the problem of measuring wear and its relationship to maintenance practice very complex. However, in order to obtain a realistic solution, certain assumptions had to be made.

These include:

- We are considering a situation outside the plant expansion or downsizing programmes. That is, there is no acquisition of new plant machinery or disposal of the old one.

- There is no change in the production procedure of the plant (i.e. no adoption of new production 
patterns, technology, etc.). The reason is that the adoption of a new production procedure will most likely evolve a more efficient method of production, consequently we have less wear. This will distort the result of the study, as the present system in place is what should be our concern.

- The plant operates on a limited number $(\mathrm{m})$ of mechanical machines. This is to restrict the size of the plant and the scope of the problems to systems that can be analyzed using mathematics of rigid and fluid mechanics (i.e. excluding electrical and chemical effects).

- The plant piping system will have its wear proportional to the rate of volume lost from leaks, joints, etc. with respect to time. The justification for this is that except using high technology principles such as intelligent pigging (used for pipeline maintenance in oil exploration companies), an easy way to observe/model wear in a pipe is to consider the rate of leakage. This can easily be modeled as a continuous function of time $v(t)$.

- That the wear in each machine has its own wear function $f(t)$ defining it. It is expressed as a continuous function of time to allow for mathematical operations of integration and differentiation to be performed easily on it. Also, each machine is allowed to have its own wear function $f_{i}(t)$ for flexibility since it will wear depending on its particular condition of operation and duration of use. Time is used as the independent variable for the wear function because wear depends on long (duration) use.

- The model will give results with units in millimeters (mm). This is assumed basically for dimensional homogeneity so that all the parameters considered will be adjusted with appropriate constants to give values of wear in the same dimension. Secondly, it is customary to measure in millimeters.

\subsection{Model development}

The model developed here considers a production plant having a number of machines on which it operates. The model can be broken down to two parts: Part $A$ and $B$. Part $A$ considers an ideal situation in which:

1. The machines are operated by the right personnel (that is, skilled manpower).

2 . The machines are operated strictly according to the manufacturer's manual.

3. Plant is given good maintenance when due.

4. Plant is loaded as specified by a manufacturer.

5. Plant is run for periods as specified by a manufacturer.

6. There is a well-stocked inventory of spare parts.
7. Running maintenance is done to avoid unnecessary down time/shut down.

8. There is before-breakdown-replacement (BBR) of worn-out parts of the plant machinery.

Part $B$ considers the effect of inefficient maintenance on the plant machinery (i.e. the additional wear occurring in the machines due to poor maintenance practice).

\section{Part A of the model framework}

In this part of the model, we assume that a plant operates ideally. That is, a good maintenance practice is in place. Let the plant machinery operate for only $t$ hours by the day. For this period, let the plant incremental wear be $\Delta w$. If the plant wear for the first day during the hours of operation is $w_{0}$, then for the second day the plant will have the wear $w_{0}+\Delta w$. On the $3^{\text {rd }}$ day, the wear would be $\left(\left(w_{0}+\Delta w\right)+\Delta w\right)$ i.e. $\left(w_{0}+2 \Delta w\right)$. For the $4^{\text {th }}$ day, the wear would be $\left(\left(w_{0}+2 \Delta w\right)+\Delta w\right)$ i.e. $\left(w_{0}+3 \Delta w\right)$. It follows that for $n$ days of operation, the plant will have the wear of $w_{n}=\left(w_{0}+(n-1)(\Delta w)\right)$.

This is an arithmetic way by which the wear of the plant increases. The justification for this is that a plant (which is composed of machines) will always have its efficiency less than one (i.e. $\eta<1$ ). This is due to the friction that comes into play when there is a relative motion between bodies. Mechanical systems are composed mainly of these linkages (having frictional forces acting). Therefore, there will be steady, though gradual wear that is here defined as $w_{n}$ by the formula for the $n^{\text {th }}$ value of an arithmetic sequence.

The graphical representation of equation (2) is presented in Fig.

It should be noted that equation (2) i.e. $w_{n}=(\Delta w)(n-1)+w_{0}$ could be compared with the general linear function of $y=m x+c$, where $c$ in the general linear equation is equivalent to the intercept (i.e. initial wear). We also note that $m$ in the general linear equation is equivalent to $\Delta w$ for the wear equa-

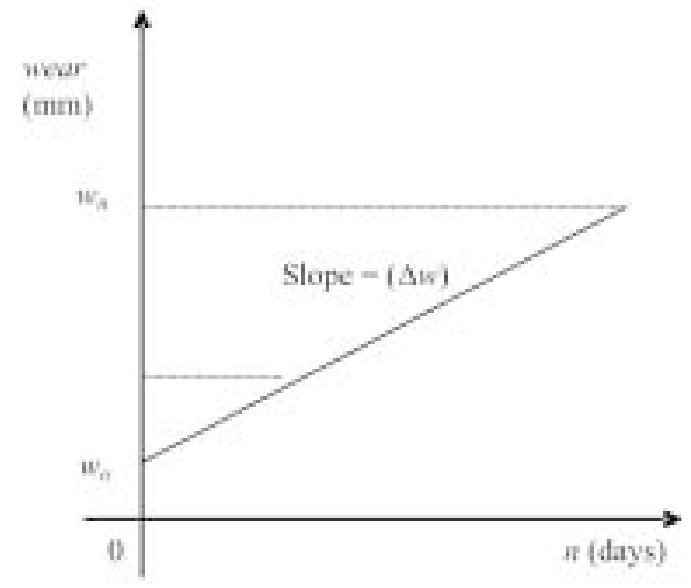

Fig. Graphical representation of equation (2) 
tion, i.e. additional day of plant operation. It should also be noted that $y=f(m)$ is the same as $w_{n}$, i.e. wear in plant after running for $n$ days. The wear graph above shows the linear variation of the wear with duration $(n)$ in days of plant operation.

\section{Part $B$ of the model framework}

This part deals with an inefficiently maintained plant. The section considers the additional wear to that in equation (2). This is due to the effect of lack of efficient maintenance of plant wear. Let us follow a stepwise approach in the development of the formula to represent this additional wear. Let the wear in each machine, say turbine, pump, compressor, etc. each be defined by their particular wear function $f_{i}(t)$ such that the total plant wear owing to these machines will be given by $w_{x}$, stated below:

$$
w_{x}=\sum_{i=1}^{m} f_{i}(t)
$$

where $m$ is the number of machines in the plant under consideration. The mean wear, which is a representative value for the plant machinery will therefore be given by:

$$
w_{y}=\frac{w_{x}}{m}=\frac{1}{m} \sum_{i=1}^{m} f_{i}(t) .
$$

Another measure of wear considered is the volume rate of leaks from joints, gaskets, or the plant piping system generally. Let the wear in the piping system be proportional to the rate of drops of volumes of the fluid conveyed with respect to time. Therefore, representing this wear by $w_{z}$ we will obtain:

$$
w_{z}=\frac{d v(t)}{d t}
$$

where $v(t)$ is the time-dependent volumetric wear function. Putting a constant of proportionality into equation (7) (for dimensional homogeneity) we will obtain:

$$
w_{z}=\frac{k d v(t)}{d t},
$$

where $k=$ wear constant for the plant piping system (network). It is important to note that wear in plant piping system depends on:

- Material of construction of the pipe.

- The material (or fluid) conveyed.

- Whether or not the pipe is submerged (subsea piping) or protected from corrosion.

Combining equations (4) and (6) to obtain combined wear $\left(w_{p}\right)$, which is expressed as:

$$
w_{p}=w_{y}+w_{z} \frac{1}{m} \sum_{i=1}^{m} f_{i}(t)+\frac{k v(t)}{d t} .
$$

In considering the effect of the individuals operating the machines, let us introduce the reciprocal $\left(\frac{1}{\alpha}\right)$ into equation (5). The range of values of $\alpha$ will depend on the expertise (training/competence level) of each machine operator (or say a mean value to represent the competence level for the plant operators). We therefore, have a new expression for wear given by:

$$
w_{Q}=\frac{w_{p}}{\alpha} .
$$

Again,

$$
w_{Q}=\frac{1}{\alpha}\left(\frac{1}{m} \sum_{i=1}^{m} f_{i}(t)+\frac{k d v(t)}{d t}\right),
$$

where $\alpha=$ manpower competence factor.

Equation (9) gives an expression that can be used to evaluate the additional wear in plant machinery due to inefficient maintenance. Adding equations (1) and (9) to obtain a general wear equation represented by $w_{R}$, we have:

$$
w_{R}=w_{n}+w_{Q},
$$

and

$$
w_{R}=\left(w_{0}+(n-1) \Delta w\right)+\frac{1}{\alpha}\left(\frac{1}{m} \sum_{i=1}^{m} f_{i}(t)+\frac{k d v(t)}{d t}\right) .
$$

Equation (10) above gives an expression that can be used to determine/evaluate plant wear. It can be viewed as a sum of:

- Normal wear (good maintenance practice): $w_{n}$.

- Additional wear (poor maintenance practice): $w_{Q}$.

\section{Case study}

Let us consider a hypothetical multinational oil and gas company with over two decades of operation in the oil rich Niger-Delta region of southern, Nigeria. This is a production plant which goal is to produce energy products such as PMS (premium motor spirit gasoline), among other petroleum derivatives. The plant operation will involve the exploration and processing of petroleum before it takes a form suitable for consumption. The plant will therefore require among other things, a complex network of piping, different machines operating at different stages of processing the crude oil. These machines involve compressors, turbines, cooling towers, throttle valves, condensers, evaporators, boilers, burners, nozzles, diffusers, etc. For the purpose of simplifying the problem, let us assume that a plant operates on only 4 machines: turbine, compressor, pump and evaporator. Let us further assume that each of these machines has its wear 
defined by a particular wear function $f_{i}(t)$ and that the wear in PPC network of the plant is defined by: $\frac{k d v(t)}{d t}$.

If the plant runs for an $8 \mathrm{hrs}$ day it has initial wear of $8 \mathrm{~mm}$. Let us further define a hypothetical range of valves for $\alpha$ for which its values will range between 0 and 1 . The values of $\alpha$ will be close to 1 for highly skilled manpower operating the plant and $\alpha$ will have values close to 0 for manpower of low skill. The value of the reciprocal $\left(\frac{1}{\alpha}\right)$ will therefore be low for highly skilled personnel and will be high for personnel with little skill. This will affect the total plant wear $w_{R}$ by the appropriate multiplier on factor with its effect least when considering highly skilled manpower. Let us have $\alpha$ defined over the range $0<\alpha<1$ as shown in Table 1 below.

Let us assume the machine wear function to be shown below (Table 2).

While $f_{i}(t)$ used here are hypothetical values actual values can be obtained in practice from manufacturers or taking data, observing relations, then generalizing to get machine wear function.

From Table 2,

$w_{y}=\frac{1}{m} \sum_{i=1}^{m} f_{i}(t)=\frac{1}{4} \cdot(179+92+357+91)=179,75 \mathrm{~mm}$.

Therefore $w_{y}=\frac{1}{m} \sum_{i=1}^{m} f_{i}(t)=179,75 \mathrm{~mm}$.

Assuming the cost volume function is given by: $v(t)=t^{2}+4 t$.

Table 1. Skill level and manpower competence factor

\begin{tabular}{|l|c|}
\hline \multicolumn{1}{|c|}{ Level of skill } & $\begin{array}{c}\text { Manpower competence } \\
\text { factor }(\alpha)\end{array}$ \\
\hline Highly skilled & $0,9<\alpha<1$ \\
\hline Semi skilled & $0,5<\alpha<0,9$ \\
\hline Unskilled & $0<\alpha<0,5$ \\
\hline
\end{tabular}

Table 2. Machine wear function

\begin{tabular}{|c|l|c|c|}
\hline$i$ & Machine & $\begin{array}{c}\text { Wear function } \\
f_{i}(t)\end{array}$ & $\begin{array}{c}\text { Duration }(t), 90 \\
\text { days }\end{array}$ \\
\hline 1 & Pump & $t+1$ & 91 \\
\hline 2 & Evaporator & $4 t-3$ & 357 \\
\hline 3 & Compressor & $t+2$ & 92 \\
\hline 4 & Turbine & $2 t-1$ & 179 \\
\hline
\end{tabular}

Therefore $\frac{d v(t)}{d t}=2 t+4$,

where $t=90$ days, $\frac{d v(t)}{d t}=2 \cdot(90)+4=184 \mathrm{~mm}^{3} /$ day.

Assuming $k=0,5$ day $/ \mathrm{mm}^{2}$.

From equation (6),

$w_{z}=\frac{k d v(t)}{d t} \mathrm{~mm}=0,5 \cdot 184=92 \mathrm{~mm}$.

Let $(\Delta w)$, incremental wear in a well maintained plant be $0,4 \mathrm{~mm}$ for an $8 \mathrm{hrs}$ day of plant operation.

$w_{0}=8 \mathrm{~mm}$ (i.e. wear after $1^{\text {st }}$ day of plant operation).

$n=$ number of day of running the plant.

$\Delta w=0,4 \mathrm{~mm}$.

It follows that:

$w_{n}=w_{0}+(n-1)(\Delta w)=8+(90-1) \cdot 0,4=43,6 \mathrm{~mm}$.

Also,

$w_{y}=\frac{1}{m} \sum_{i=1}^{m} f_{i}(t)=179,75 \mathrm{~mm}$,

$w_{z}=\frac{k d v(t)}{d t}=92 \mathrm{~mm}$.

Also,

$w_{\alpha}=\frac{w_{y}+w_{z}}{\alpha}=\frac{1}{\alpha}\left(\frac{1}{m} \sum_{i=1}^{m} f_{i}(t)+\frac{k d v(t)}{d t}\right)=$

$\frac{1}{\alpha}(179,75+92)=\frac{271,75}{\alpha}$.

We also note that

$w_{R}=\left(\frac{271,75}{\alpha}+43,6\right) \mathrm{mm}$.

Now consider the level of skill (expertise) of the operators of the plant machinery:

- Highly skilled manpower.

Let $\alpha=0,97$, then

$w_{R}=\frac{271,75}{0,97}+43,6=323,75 \mathrm{~mm}$.

- $\quad$ Semi-skilled manpower.

Let $\alpha=0,58$, we also have

$w_{R}=\frac{271,75}{0,58}+43,6=512,13 \mathrm{~mm}$.

- Unskilled manpower.

Let $\alpha=0,2$, we also have

$w_{R}=\frac{271,75}{0,2}+43,6=1402,35 \mathrm{~mm}$. 


\section{Analysis of the results}

From the case study considered the wear in a wellmaintained plant $w_{A}$ was $43,6 \mathrm{~mm}$ when the plant was run for the duration of 90 days. For which the plant operates only $8 \mathrm{hrs}$ of each working day. The effect of bad maintenance practice on the plant over the same duration gave the following results. For highly skilled personnel operating the plant i.e. assuming a manpower factor of (say $\alpha=0,97$ ) the plant wear was $323,75 \mathrm{~mm}$; for semi skilled manpower (say $\alpha=0,58$ ) the plant wear obtained was $512,13 \mathrm{~mm}$ while for unskilled manpower a value of $1402,35 \mathrm{~mm}$ was obtained for running the plant for the same 90 day duration (at $8 \mathrm{hrs}$ for each day). $8 \mathrm{hrs} /$ day is the actual working, running time assumed for the plant in this case study.

Hypothetical values were used to test the mathematical behavior of a part and the entire model as shown below:

- The wear in a well maintained plant was given as $w_{n}=\Delta w(n-1)+w_{0}$ (equation 1$)$ representing $w_{n}, \Delta w,(n-1)$ and $w_{0}$ by $y, m, x$ and respectively.

Equation (1) can be rewritten as shown below: $y=m x+c$.

This gives the equation for a linear graph where $m=\Delta w$ represents the scope of the graph. From the case study considered, the following values were utilized.

$m=\Delta w=0,4 \mathrm{~mm} /$ day, $w_{0}=c=8 \mathrm{~mm}$.

By substituting this in the expression for $y$ above, $y=0,4 x+8$. From here, we could construct Table 3 .

- $\quad$ Still working on ideally-maintained plant which wear was modelled as: $w_{n}=\Delta w(n-1)+w_{0}$ (see equation 1).

Assuming now that the initial wear recorded in the plant after the first day of operation (i.e. $w_{0}$ ) was the same as the incremental wear $(\Delta w)$ such that:

$$
w_{0}=\Delta w .
$$

Table 3. Mathematical relationship among $n, x$, and $w_{n}$

\begin{tabular}{|c|c|c|}
\hline$n$, days & $x=(n-1)$, days & $w_{n}, \mathrm{~mm}$ \\
\hline 6 & 5 & 10 \\
\hline 11 & 10 & 12 \\
\hline 16 & 15 & 14 \\
\hline 21 & 20 & 16 \\
\hline 26 & 25 & 18 \\
\hline 31 & 30 & 20 \\
\hline 36 & 35 & 22 \\
\hline 41 & 40 & 24 \\
\hline
\end{tabular}

The above equation for $w_{n}$ could be re-written as $w_{n}=\Delta w(n-1)+w$. This means the same as $(\Delta w) n=\Delta w+\Delta w$. If $w_{n}, \Delta w$ and $n$ are replaced by $y, m$ and $x$ we will obtain $y=m x$. This is another linear graph for which y intercept (i.e. $c$ ) is equal to 0 . This means the plant can be viewed as having zero initial wear. From the case study, $m=\Delta w=$ $0,4 \mathrm{~mm} /$ day. Therefore, $y=0,4 x$. The behaviour of the values is shown in Table 4 .

For part $B$ (additional wear), we refer to equation (6):

$$
w_{z}=\frac{k d v(t)}{d t}
$$

This equation gives the wear in the plant piping system. The volumetric wear function $v(t)$ used in the case study was $v(t)=\left(t^{2}+4 t\right)$ such that $w_{z}=\frac{k\left(t^{2}+4 t\right)}{d t}$. Therefore $w_{z}=k(2 t+4)$, where $k=$ plant piping wear constant. Consider different values of $k$ depending on:

- Corrosion prevention.

- $\quad$ Fluid conveyed.

- $\quad$ Condition of pipe use (surface/submerged piping).

- $\quad$ Process effects e.g. turbulence.

The value of $k$ assumed in the case study is $0,5 \mathrm{day} / \mathrm{mm}^{2}$. Assuming this first consideration, that of all the (4) factors listed above corrosion prevention has the greatest net effect, the value of $k$ will therefore be reduced as applying corrosion prevention will prolong pipe life and therefore reduce its wear.

By assuming $k=0,2$ day $/ \mathrm{mm}^{2}$,

$w_{z 1}=0,2(2 t+4)=(0,4 t+0,8)$. The values obtained here could be put into Table 5 .

- Consider the effect of the fluid conveyed on the plant piping. Let the fluid conveyed in most of the pipe network be heavy oil. This will give the pipe a protective covering within as a film of oil will always remain on the pipe surface within. $k$ will therefore be $w_{w}$. Assuming value of $k=0,1$ day $/ \mathrm{mm}^{2}$, we have:

$w_{z 2}=0,1(2 t+4)=(0,2 t+0,4)$. The values obtained here could be put into Table 6 .

Table 4. $n$ versus $w_{n}$

\begin{tabular}{|c|c|}
\hline$n$, days & $w_{n}, \mathrm{~mm}$ \\
\hline 6 & 2,4 \\
\hline 11 & 4,4 \\
\hline 14 & 5,6 \\
\hline 21 & 8,4 \\
\hline 26 & 10,4 \\
\hline 31 & 12,4 \\
\hline 36 & 14,4 \\
\hline
\end{tabular}


- Consider the effect of the process on pipe wear. Assuming for most of the network, the process involved causes a lot of turbulence in the flow. The formation and collapse of cavitation bubbles will lead to accelerated pipe wear. The value if $k$ will therefore be high for this situation. Let $k=0,65 \mathrm{day} / \mathrm{mm}^{2}$. The values obtained here could be put into Table 7 .

Table 5. $t$ and $w_{z 1}$

\begin{tabular}{|c|c|}
\hline$t$, days & $w_{z 1}, \mathrm{~mm}$ \\
\hline 5 & 2,8 \\
\hline 10 & 4,8 \\
\hline 15 & 6,8 \\
\hline 20 & 8,8 \\
\hline 25 & 10,8 \\
\hline 35 & 12,8 \\
\hline
\end{tabular}

Table 6. $t$ versus $w_{z 2}$

\begin{tabular}{|c|c|}
\hline$t$, days & $w_{z 2}, \mathrm{~mm}$ \\
\hline 5 & 1,4 \\
\hline 10 & 2,4 \\
\hline 15 & 3,4 \\
\hline 20 & 4,4 \\
\hline 25 & 5,4 \\
\hline 30 & 6,4 \\
\hline
\end{tabular}

From the general wear equation for the plant (i.e. equation 10), we have:

$$
w_{R}=\left(w_{0}+(n-1) \Delta w\right)+\frac{1}{\alpha}\left(\frac{1}{m} \sum_{i=1}^{m} f_{i}(t)+\frac{k d v(t)}{d t}\right) \text {. }
$$

In the case study, we had the following values:

$$
w_{R}=43,6+\frac{1}{\alpha} 271,75 \text {. }
$$

This gives an equation with $w_{R}$ and $\alpha$ as the only variables. Where $\alpha$ is a manpower competence factor. Plotting $w_{R}$ against $\alpha$ for the range of $\alpha$ (i.e. different levels of expertise) we use the values in Table 8 below.

Table 7. $t$ versus $w_{z 3}$

\begin{tabular}{|c|c|}
\hline$t$, days & $w_{z 3}, \mathrm{~mm}$ \\
\hline 5 & 9,1 \\
\hline 10 & 15,6 \\
\hline 15 & 22,1 \\
\hline 20 & 28,6 \\
\hline 25 & 35,1 \\
\hline 35 & 41,6 \\
\hline
\end{tabular}

Table 8. $\alpha$ versus $w_{R}$

\begin{tabular}{|c|c|}
\hline $\begin{array}{c}\text { Manpower competence } \\
\text { factor } \alpha\end{array}$ & Plant wear $w_{R}, \mathrm{~mm}$ \\
\hline 0,1 & 2761,0 \\
\hline 0,2 & 1402,35 \\
\hline 0,3 & 949,43 \\
\hline 0,4 & 722,98 \\
\hline 0,5 & 587,10 \\
\hline 0,6 & 496,52 \\
\hline 0,7 & 431,81 \\
\hline 0,8 & 383,28 \\
\hline 0,9 & 345,54 \\
\hline
\end{tabular}

\section{Conclusions}

From the foregoing, it can be seen that normal wear $w_{n}$ is associated with every plant. This wear however, is shown in growth and can therefore be modeled/represented mathematically using an arithmetic sequence. Its value is small compared to the additional wear in plant machinery when it is neglected (poor maintenance practice). Having machines operated by well trained/highly skilled personnel is also a very important maintenance strategy as the resulting accelerated wear of using unskilled manpower is obvious from the model results.

\section{References}

1. Calmer, H. An investigation of the wear behaviour of ceramic coated metals. MSc Thesis. Erciyes University, Turkey, 2002.

2. Santarisi, N. S.; Almomany, R. M. Mathematical modeling of wear rate of non-reparable parts and their replacement strategies: cement mill liners as a case study. Journal of Quality in Maintenance Engineering, Vol 11, No 1, 2005, p. 68-81.

3. Waara, P. Lubricant influence on flange wear in sharp railroad curves. Industrial Lubrication and Tribology, Vol 53, No 4, 2001, p. 161-168.

4. Luis, C. J.; Puertas, I.; Villa, G. Material removal rate and electrode wear study on the EDM of silicon carbide. Journal of Materials Processing Technology, Vol 164/165, 2005, p. 889-896.

5. Karamis, M. B.; Yildizli, K.; Cakirer, H. Wear behaviour of Al-Mo-Ni composite coating at elevated temperature. Wear, Vol 258, 2005, p. 744-751.

6. Danks, D.; Clayton, P. Comparison of the wear process for eutectoid rail steel: field and laboratory tests. Wear, No 120, 1987, p. 233-50.

7. Danks, D.; Clayton, P. Result of rail wear test at FAST, Vol 1: FAST/HAL test summaries. AAR Research Review, 1987.

8. Kumar, S.; Alzoubi, M. F.; Alsayyed, A. Wheel rail adhesion wear investigation using a quarter scale laboratory testing facility. ASME Winter Annual Meeting, 1995. 